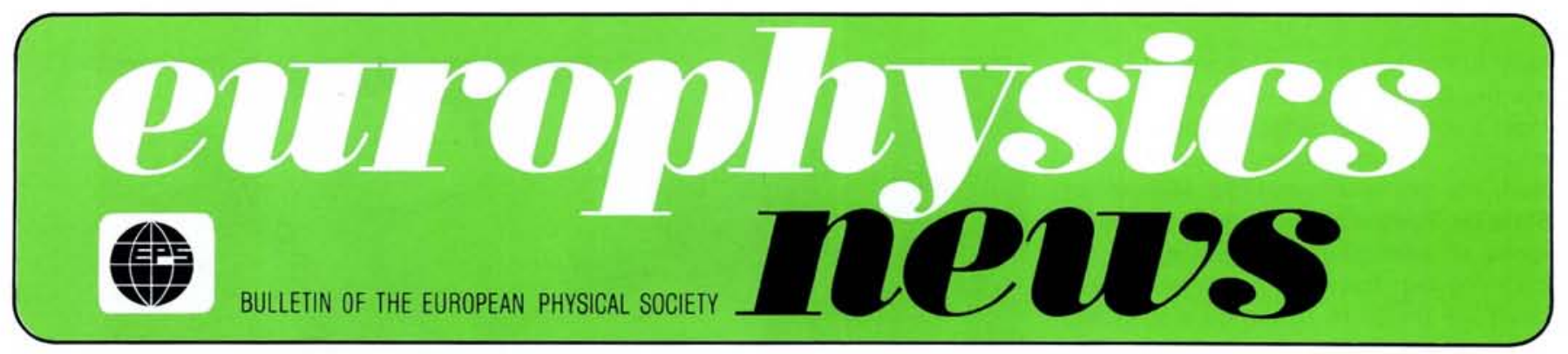

J.A. Volume $12 \quad$ Number 10

October 1981

\title{
Impressions of Istanbul
}

On the tranquil and picturesque campus of Bogaziçi University, set on a green plateau overlooking the busy Bosphorus Straits, some 650 physicists from all over Europe and the USA explored the many fascinating areas of physics that are currently in rapid evolution. The setting, with the separate buildings of the University grouped round a central square was ideal; as much physics was discussed outside the lecture halls as within. In the main theatre where the plenary sessions were held, the loud-speaker system was impeccable and from every part of the room the lecturers were clearly audible. The seminars were in smaller, more intimate rooms, adapted to the personal interaction that was to be expected.

The Society had decided to hold its 5 th General Conference in Istanbul partly because it had been convinced that the University and the Turkish authorities would do all in their power to make the Conference a success. In this they amply fulfilled the expectations. Professor Inönü had worked tirelessly to see that everything went smoothly and he was backed by a University team that combined adaptability with energy and good humour. Mention must be made especially of Professor
Akyüz who at all hours of the day - and long into the evenings - could be found busy on the campus, prepared to put his hand to anything: move tables, distribute papers, set up microphones etc., a personal tour de force that was appreciated by everyone.

Even the weather conspired to keep the conference halls full - sunny and calm before and after, dull and stormy during the week. And full they were. Hardly a plenary session, evenings included, was not packed and the quality of the lectures justified the attention. Congratulations to the speakers and to the Programme Committee under E. Matthias and W. Merz that invited them. For the most part their subjects were clearly set out and pitched at a level suited to the non-specialist. Pride of place must however go to D.K. Scott of Michigan who enthralled his audience with his exposé on the richness of the configurations of nuclei -300 naturally stable, 1600 radioactive that are accessible and a further 6000 waiting to be discovered. Uninhibited in his approach - perhaps through having lost his luggage between Michigan and Frankfurt - he demonstrated the value of professionalism in presentation. A touch of humour to relax the ten-

A typically packed session; the chairmen of the International Programme Committee, E. Matthias and $W$. Merz are fourth from the right and left respectively.

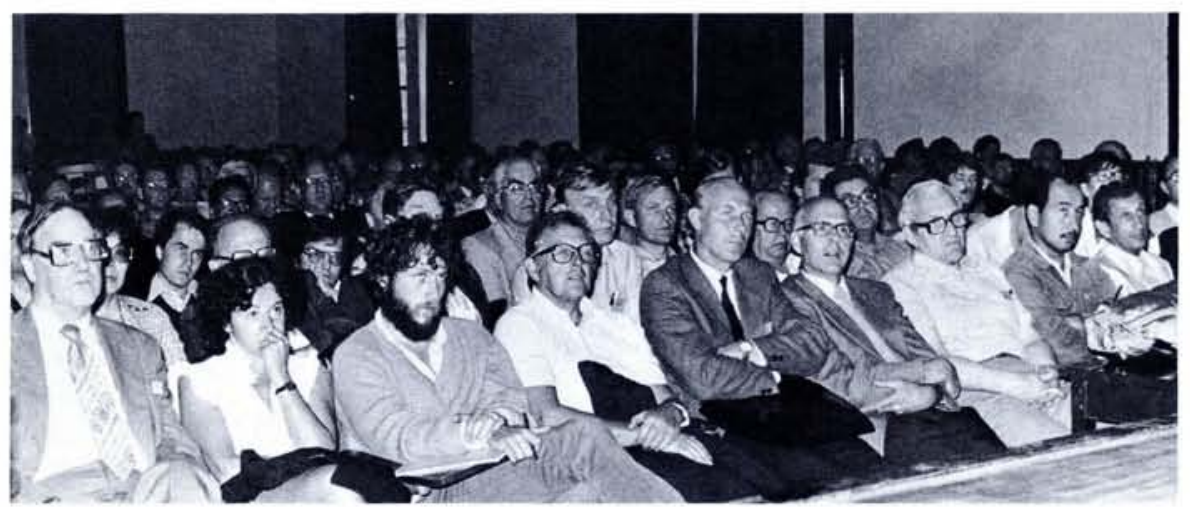

sion, some nice aphorisms to stress his points and at the end a glimpse of the Eldorado to which nuclear physicists are heading.

Few questions followed the plenary sessions but this was due to the sense of repleteness that most inspired, and also the amount of information that most contained. Doubts are always voiced about the usefulness of a general conference and one could note that the majority of participants attended the seminars that were on subjects in their own field, but there was much physics learned in the plenary sessions and many participants expressed surprise at the intensity of interest they generated. It is easy to forget how recently some fields have opened and how rapidly others develop. For example, it is only 15 years since we have been able to detect interstellar molecules which is the very stuff of which we are made (after a certain evolution) and only now are we able to explore the Universe over the whole EM spectrum.

A second reason for choosing Istanbul was the contribution that the Conference could make to Turkish physics. Over 200 physicists from Turkey took part and there is reason to believe that the Conference will mark a new departure in the country. Turkey is currently celebrating the 100th anniversary of the birth of Kemal Atatürk and many references were made to his

\section{Contents}

Impressions of Istanbul 1

Council

General Meeting

Inauguration of EISCAT

Surface Structure Investigations

Using He Diffraction

The Volcanism of lo

Problems of Physics in the

Developing Countries of Europe

11 
belief in the importance of science to combat the fanatic way of thinking - "The most truthful and perfect guide in life is science". Special stamps were printed to mark the occasion* and the Minister of State for Science at the opening ceremony spoke of science and technology as the main driving force for change. Turkey faced the problems of the developed and underdeveloped countries at the same time, but the challenge was accepted and present plans called for expenditure on R \& $D$ in the country to be raised from the present $0.2 \%$ to $1.0 \%$ GNP by 1990 .

An immediate benefit to the University was a significant addition to the physics library as a number of publishers, notably North Holland and Pergamon Press, who had mounted an exhibition of books on the campus, presented their collections to the University at the end.

Was it imagination, or is there a new mood amongst physicists that is less pessimistic than a few years ago - less tendency to bewail the declining interest in physics or the lack of jobs or the impact of science on our way of life and more a desire to recognize the boundary conditions and make the best of what was possible? This was certainly evident in the plenary sessions and seminar devoted to the impact of very large scale integration of electronic functions and the invasion of microelectronics.

Economic power, it was suggested, was now measured by a capacity for chip building, as against ship building in the past, and the market was already such that 10000 tons of silicon were used last year for making integrated circuits. A whole new jargon had been invented which was difficult to keep up with and a whole new field of physics would have to be developed in order to keep pace with device development. We have now reached the stage when the number of electrons taking part in functions was below the level at which statistical mechanics any longer applied and devices were being built which currently defied a convincing explanation.

The rate of development had been enormous, with the number of functions that could be built into a single chip going up by 100000 in 20 years with costs per function coming down by the same factor. The curve had to flatten out at some time but there was no sign yet. Disagreements were evident on the role of microprocessors and the degree to which they would become generalized and then dedicated to particular applications. There was little doubt however that their impact on society would be felt in all sectors and would entrain a major shift in skills and occupations. Locally, the immediate effect could be negative, but overall there was reason to believe that

- Envelopes carrying the stamps and franked on the day of issue are available at the EPS Secretariat, price Sw.Fr. 5.-

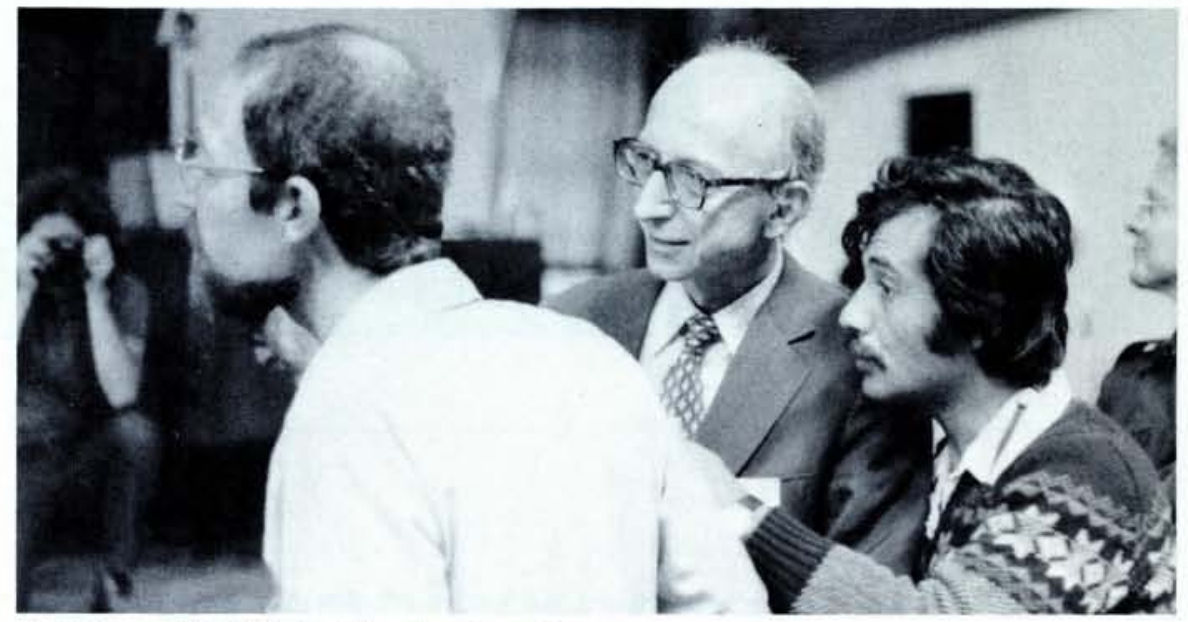

A. Salam and V. Weisskopf in relaxed mood.

the balance could be positive if institutions - not least our schools and universities were sufficiently adaptable. Physicists had a major role to play, as they were involved from the very beginning at the materials level, through to the design and operation of complete systems.

They could not do everything however, and a stirring call was made to the scientific community to come together to solve the problems of converting solar power on a massive scale. Heterosemiconductors it was claimed, had now reached a level of efficiency of photo conversion $160 \%$ was quoted) that justified large-scale exploitation. What was needed was a massive international effort on producing these materials economically and devising practical concentrators and storage systems.

\section{Weisskopf and Salam}

It was a great disappointment that at the last moment P.A.M. Dirac was prevented by ill health from coming to Istanbul, but an overflow audience were treated to two brilliant talks by V. Weisskopf and A. Salam, the first tracing the development of our concepts of fields and particles since Maxwell, and the second looking ahead to the prospects for further unification by extrapolating our present ideas.

Whilst acknowledging the tremendous strides that physics had made this century and referring to Dirac's contribution of

1927 as "a triumph of theoretical physics" and quoting Dirac's own words that "my equation is more intelligent than I am", Weisskopf refused to rest on our laurels and itemized a number of pressing unknowns.

1) Is field theory procedure really sound and does it apply also to large couplings? Field theory is a description of a system with an infinite number of degrees of freedom, but we have a mathematical understanding of coupled systems only when there is a finite number as in quantum mechanics. Even moving from the hydrogen atom with three degrees of freedom to the helium atom with six, introduced great complexities, and it is just not known how far the Schrödinger equation can be pushed. On the other hand if the coupling between particles is very weak, we are able to arrive at a global description by considering the interaction between just two particles and establishing a hierarchy of corrections. This has been very successful in quantum electrodynamics where the gyromagnetic ratio of the muon has been calculated to the 10th decimal place - just because the fine structure constant is so weak. But in circumstances where the coupling is strong, the situation might be very different.

2) What do we understand by fluctuations in the vacuum? New concepts are

\section{E. Inönü (centre) and R.O. Akyüz (left) with a colleague check final details.}

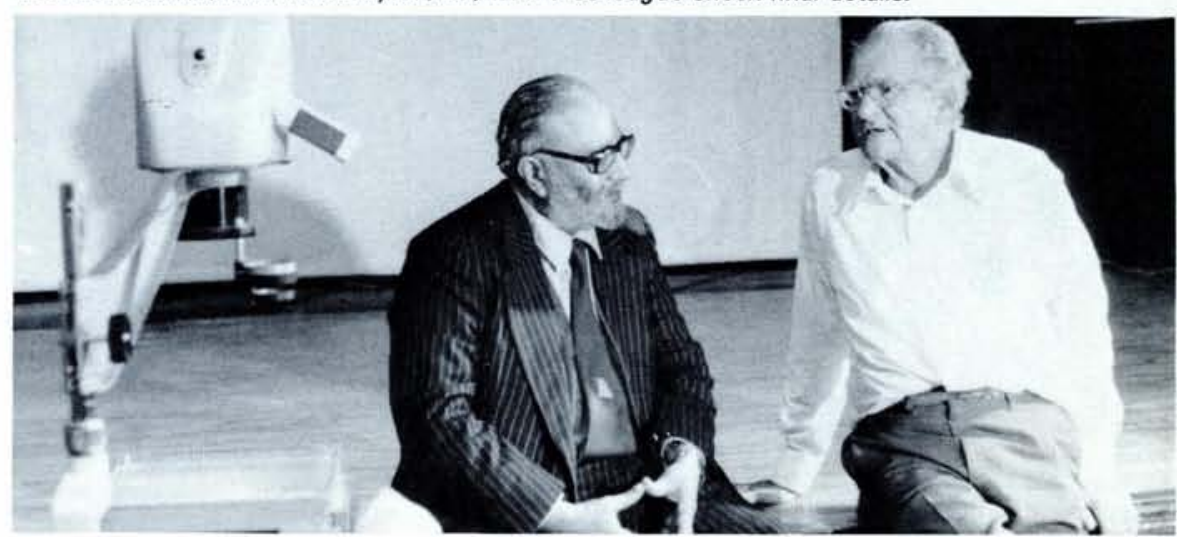


needed to cope with the notion of a vacuum that is totally populated. The only way we can talk about the vacuum today is as the lowest state of energy and what we measure in a system is the energy with respect to this lowest state, but new semantics have to be invented.

3) Is Quantum Electroweak Dynamics correct? The introduction of the Higgs mechanism which allows originally massless vector mesons to acquire a mass, as massless scalar mesons disappear, introduces uncomfortable consequences when gravity is introduced. It is difficult then to avoid Einstein's cosmological constant which has been proved not to exist.

4) Why have the fundamental constants the values they have? Why for example should the ratio between the masses of the proton and electron - the basis of all matter and life - be about 1836 ?

5) Why should almost the whole Universe be able to operate with only simple quarks? Are we being unnecessarily complicated in introducing other variants with such whimsical names?
6) Alternatively is there any end to the quantum numbers that are necessary to describe our Universe?

Salam declined to provide simple answers to these questions and instead addressed himself to the evolution of our concept of charges and the prospect of a unification of the electronuclear charge with gravitational charge, and what could be expected from experimentation at higher energies. On the very far horizon was the Planck mass of $10^{19} \mathrm{GeV}$ equivalent to a distance of $10^{-33} \mathrm{~cm}$ where space is so curved that our classical concepts of space and time are bound to fail.

Our more modest experience with current accelerators had allowed us to take a firm step on the road to unification and led us to formulate strategies for the immediate future based on the success of symmetries and gauge interactions. A crucial test will be to check on the existence of the $\mathrm{W}$ boson which mediates the weak interaction, and the $Z^{\circ}$ boson, and these should be revealed by the proton-antiproton experiments now under way at CERN.

\section{Council}

In reviewing the progress of the Society during the past months, the President reported on the steps that had been taken to enlarge the Society's contacts with other organizations concerned with physics in Europe and the world at large. EPS was recognized as one of the most successful societies for the promotion of scientific collaboration in Europe.

\section{External Relations}

Both IUPAP and the European Science Foundation had expressed a wish to support our activities. For example, the ESF was mainly concerned with identifying areas where collaborative programmes in science could increase the efficiency of research. This is done by bringing dispersed groups together and promoting a rational use of research facilities. It was interested in drawing on the expertise that was assembled within the EPS Divisions and in a collective approach towards promoting multi-national projects in physics. Although the ESF was not itself a fund granting agency, it had an efficient system for mobilizing the resources of the main western European funding organizations.

In the past, the notion of a world physical society had been put forward, but the complexities and costs of such an enterprise seemed prohibitive and present thinking lay more in the direction of an informal collaboration between the big physical societies. Encouraging exchanges had already been held with the American Physical
Society and these would be pursued as a pilot operation which could be followed by comparable links with the Japanese and other major regional physical societies.

In an initial phase one could envisage an exchange of privileges between members of EPS and APS, for example in the sponsoring of papers at conferences (see below) and in charges for certain publications. The European Journal of Physics has already been offered in the USA at the same prices that were charged to European physicists and the APS had offered Rev. Mod. Phys. to EPS members at the US rate. Other exchanges could follow in the future.

Another move that has been proposed, and which Council approved, is that a new category of membership should be created which would allow members of the APS, not resident in Europe, to become members of the EPS for an annual subscription approximately equivalent to five unit fees - the logic being that this was equivalent to the total income to the Society from a $4 \mathrm{C}$ Member. This would require a change in the Statutes and the Executive Committee will be formulating a specific proposal in time for Council to take a formal vote at its next meeting on 1-2 April 1982 in Geneva.

Discussions were continuing with the various optical societies in Europe, notably with the International Commission of Optics of IUPAP and the European Optical Committee, and it could be hoped that within a few months, European optical
By the end of the century it can be expected that centre of mass energies of $10^{4}$ $\mathrm{GeV}$ will have been reached and beyond that, laser accelerators with a capability of $2 \mathrm{GV} / \mathrm{m}$ could push the energy to $10^{5} \mathrm{GeV}$. At these energies, the lepton transformation of the proton could be observed if the life time of the proton is under $10^{32}$ years. On the other hand, grand unification theory indicates that it is not until $10^{14} \mathrm{GeV}$ that all the coupling parameters will have come together and have the same strength. At this point all matter will be the same. It seems improbable nevertheless that along the way to this energy Nature will not have new surprises in store.

Salam from his special position as Director of the International Centre for Theoretical Physics in Trieste had also a clear message to give on the need to help the less fortunate countries, to build up their skills and their confidence. His concern with unification does not end with theoretical gauge theories, but extends to symmetries that we hope to establish in our own physical world.

\section{EPS Travelling Lecturer}

The accolade of 1982 EPS Travelling Lecturer has been awarded to Professor Isaac F. Silvera from the University of Amsterdam. Awarded as a sign of recognition of outstanding achievements in physics, it also provides, through a lecture tour, a means for communicating new results in physics in a number of EPS countries.

The very interesting results recently obtained by Professor Silvera on atomic hydrogen and deuterium at high pressures make such a tour particularly appropriate.

Professor Silvera has undertaken to visit a number of countries, including Scandinavia, France, Switzerland and Italy in the Spring of 1982.

physicists could be drawn within the fold of the EPS. One could also note the growing relations with UNESCO - for example the seminar in Istanbul (see page 11) and a seminar for editors and publishers of physics journals in Europe that would take place next January. There was also to be a meeting in Trieste on 24-26 September at the ICTP on science transfer, at which EPS would be represented, particulary in the round table session on the role to be played by societies such as ours.

\section{Associate Members}

There was a general desire to see more attention being paid to our associate mem- 